\title{
GUERVÓS, J., FERNÁNDEZ, J. (2017): FUNDAMENTOS PARA LA ENSEÑANZA DEL ESPAÑOL COMO 2/L. EDITORIAL ARCO/LIBROS-LA MURALLA, S.L.: MADRID.
}

\author{
María Gabriela Amador Solano \\ Instituto Tecnológico de Costa Rica \\ gamador@itcr.ac.cr
}

El libro reúne los fundamentos teóricos, metodológicos y didácticos de la enseñanza de L2/LE, cuyo público meta es el profesorado en formación. Sus autores, Javier de Santiago Guervós y Jesús Fernández González tienen una vasta experiencia como docentes en el campo, por lo que consideraron necesario realizar esta obra para proporcionar una visión panorámica, accesible e interesante que permita planificar la docencia. Ambos profesores centran sus investigaciones en la lingüística aplicada a la enseñanza de lenguas y han impartido cursos de formación en distintas instituciones españolas y extranjeras.

La obra está organizada en 25 unidades que desarrollan con profundidad aspectos de la lingüística aplicada a la enseñanza del español L2 (segunda lengua) / LE (lengua extranjera), así como la metodología de su didáctica. Los autores sugieren que se use para un curso completo, o, de manera modular, para contextos específicos. Sin duda el texto abarca todas las áreas que un educador debe conocer para adentrarse al mundo de la enseñanza/aprendizaje del español como L2/LE. Conviene tenerlo en las bibliotecas de cada centro de enseñanza, como consulta o bien para refrescar o reforzar conocimientos ya adquiridos, completar o complementar aquellos sobre los que se tenga una visión más superficial, y acercarse a otros que quizá no se haya tenido oportunidad de trabajar adecuadamente.

El primer capítulo, llamado El español en el mundo, expone a través de ejemplos que no es fácil establecer los límites en los que empieza y acaba una lengua. Señala como observaciones interesantes que un gran número de hablantes utiliza habitualmente más de una lengua, a pesar de que la distribución geográfica sea desigual. Al avanzar en el texto de este primer abordaje, el lector puede reflexionar sobre las distintas posiciones que ocupan las lenguas del mundo, en orden de importancia, a partir de los indicadores que se consideren: geolingüísticos, económicos, científicos o culturales.

Por otro lado, el panorama que nos brinda este capítulo acaba profundizando en el interés que despierta aprender una lengua como L2/LE y el poder acercarse a la cultura o culturas en las que esa lengua se inscribe. Bajo este indicador, entre los idiomas más estudiados en

Para citar esta reseña / To cite this book review: Amador Solano, María Gabriela (2018). Reseña de Guervós, J., Fernández, J. Fundamentos para la enseñanza del español como 2/L. Madrid, Arco/ Libros-La Muralla, 2017. ELUA, 32: 369-374. doi: 10.14198/ELUA2018.32.17

Enlace / Link: http://dx.doi.org/10.14198/ELUA2018.32.17 
el mundo como lenguas extranjeras, el español ocuparía el tercer lugar. Los resultados de estadísticas sobre este tema los brinda el Instituto Cervantes, Berlitz, Golberg et al, ACTFL y Eurostat; a pesar de ello, se aclara que falta mucha información para trazar una panorámica completa de la situación y casuística del aprendizaje de E/LE en el mundo.

El español como tal, según los autores, se caracteriza por tener contacto constante con otras lenguas, lo cual propicia el desarrollo de diversas variedades dialectales y sociodialectales. A la vista de este panorama, el libro nos hace reflexionar sobre cómo enfocar la enseñanza del español como L2/LE y plantea en primer lugar, el exponer a los aprendices a las distintas variedades, lo cual se puede lograr a través del acceso a las tecnologías. En segundo término, el docente debe usar su propia variedad dialectal para asegurar su comprensión ante el alumnado. Por último, a modo de sugerencia, proponen reflexionar con el educando sobre los diferentes usos dialectales e ilustrarlos con numerosos ejemplos para brindarles una visión abierta y comprensiva del español.

En el segundo capítulo, Los contextos de aprendizaje, se insiste en la siguiente idea: la mejor forma de aprender un idioma es en el propio contexto, pues se multiplica la oportunidad de acceder a una gran cantidad de input lingüístico. En este caso, los autores distinguen entre el aprendizaje de una segunda lengua (L2) y la lengua extranjera (LE). La primera se lleva a cabo en el entorno social de la lengua nativa, el caso de un residente alemán que estudia español en España. La segunda, en un contexto académico, en el país de origen, caso de un alumno francés que estudia el español en una escuela de Francia. Sin embargo, puede haber un contexto mixto, en el que se interaccione entre lo académico y lo natural, por ejemplo, un estudiante canadiense de español que asiste a clases, durante una estancia en Argentina.

Bajo el concepto y características de un inmigrante, en el caso del español, la problemática lingüística y cultural derivada de la inmigración comenzó a vivirse en España de manera notable a partir del año 2000. Para el 2016 hay una necesidad de facilitar el aprendizaje del español a inmigrantes adultos y la de compensar las dificultades lingüísticas de los hijos de los inmigrantes en su proceso de escolarización. En los diferentes programas, existen factores que influyen en el rendimiento lingüístico de una estancia en el extranjero. Conviene leer el capítulo dos para analizar aspectos que generalmente inciden en el aprendizaje del idioma, por ejemplo, la socialización e integración con nativos. Se demuestran estudios de investigación en los que se comprueba que estos procesos facilitan la comunicación y adquisición de la lengua meta. En teoría, los objetivos de la estancia en el extranjero son, por orden de importancia: el progreso lingüístico, el conocimiento de la cultura del país y el recorrido turístico dentro y fuera del país.

Por su parte, los capítulos dos y tres abordan la adquisición bilingüe en la primera etapa de vida y su desarrollo en contextos naturales y académicos. Es un tema de suma importancia cuando se trabaja en la enseñanza de una segunda lengua, pues como afirman los autores, se deben tener claros los objetivos en cada contexto. Por ejemplo, en la escuela bilingüe donde aceptan en su mayoría niños monolingües, cuyos padres quieren que aprendan otra lengua, no se recibe el mismo estímulo que en un contexto natural, donde se activa el entorno lingüístico dentro del desarrollo biológico de un ser humano. En este sentido, los distintos niveles (fónico, léxico-semántico, gramatical y pragmático) van asumiendo un rol fundamental en la adquisición del idioma.

De esta manera, conforme leemos vamos entendiendo a través de los estudios que los niños siguen un camino muy parecido en la adquisición de la L1, en un ambiente sin ningún 
tipo de enseñanza formal, sin reglas gramaticales ni prejuicios por usar tal o cual secuencia de oraciones. En L2/LE, en cambio, se observa una gran variación porque el educando se enfrenta a preocupaciones por el uso de la gramática y de un determinado dialecto según la región. Existe entonces mayor consciencia de la lengua y de factores que puedan influir en su progreso. Basado en estas premisas, el capítulo cuatro deja claro que la adquisición temprana de una segunda lengua supone una mayor garantía de alcanzar un nivel nativo o aparentemente nativo. Para profundizar en el tema se comentan una serie de hipótesis explicativas de la adquisición de L2/LE, con mención de teorías como el análisis de errores, estudios de secuencias de morfemas y construcciones sintácticas, modelo del monitor, aculturación y el modelo de la interlengua.

En el avance de la lectura del libro se puede notar la inclusión de la bibliografía que a través de las décadas recoge la investigación en el campo de la enseñanza de L2/LE. A partir de finales de la década de los ochenta, los estudios de adquisición en esta área se multiplicaron y con ellos el número de propuestas teóricas. Se distinguen enfoques como el lingüístico, cognitivo, interaccionista, sociolingüístico y sociocultural. A su vez, estos incluyen teorías como la del procesamiento del input, de la procesabilidad, adquisición de destrezas, entre otras. Lo cierto es que surge de todas ellas un desafío por crear una teoría que unifique y concretice, pues al analizarlas, se marcan tantas diferencias que parecieran piezas separadas. Independientemente de la teoría que se adopte, los autores aconsejan exponer a los aprendices al mayor número posible de variedades regionales para que desarrollen una sensibilidad dialectal y sean capaces de entender a los hablantes de diferentes zonas del dominio lingüístico.

Entre los aspectos valiosos de este libro, del capítulo cinco al 12 se hace referencia a definiciones, características, ejemplos, sugerencias y ejercicios para cada una de las siguientes competencias: comunicativa, psicolingüística, contraste interlingüístico, competencia estratégica, sociocultural, gramatical, fónica y léxico-semántica. Dominar una lengua, por tanto, implica conocer estructuras, contextos culturales, explicaciones léxicas, interpretaciones de uso, desarrollo del sistema cognitivo, codificación semántica y fonológica.

Estos primeros doce capítulos permiten reflexionar sobre cómo los hablantes están inmersos en unas coordenadas culturales y tienen un conocimiento del mundo que sirve de marco para la comunicación. Una de las principales dificultades en la producción del lenguaje en una L2/LE es la transición del pensamiento y planificación a la codificación gramatical y semántica. La tentación inicial y persistente es utilizar los de la lengua materna.

Otro punto importante por mencionar en esta reseña es la cantidad de sugerencias que dan los autores para el desempeño del docente cuando trabaja en la enseñanza de L2/LE. Por ejemplo, en el capítulo ocho, sobre la competencia léxico-semántica se enfatiza en que el educador debe preocuparse por realizar actividades que respondan al vocabulario usado por los aprendices. Un investigador muy valorado en este campo es Nation (2013), el cual sugiere leer la misma historia varias veces a un niño o en el caso de los adultos, se recomienda un libro por capítulos, para que el vocabulario se repita y así retenga con mayor facilidad la escritura y la interpretación.

Mientras tanto, en el capítulo nueve, se explica la competencia gramatical y la necesidad de enseñarla en contexto para activar el proceso comunicativo. Resulta interesante en esta parte, el contraste interlingüístico mostrado para ilustrar diferencias en morfología, esquemas sintácticos y distinciones semánticas, con el fin de entender cuál es la perspectiva desde 
la que parten los aprendices y cómo la comparación ayuda a simplificar la comprensión de los problemas gramaticales. Se hace alusión a las variantes que se le han hecho a lo largo de la historia a uno de los modelos de la presentación explícita de la gramática, el de las 3 P: presentación, práctica y producción.

Dominar una lengua no es solo conocer su vocabulario o sus estructuras gramaticales, es saber qué vocabulario y qué estructuras son adecuadas según el contexto y la intención. De ahí la importancia de la competencia comunicativa, presentada en el capítulo diez. Esta va de la mano de la competencia sociocultural, pues para comunicarse en una sociedad específica se debe hacer dentro de sus valores culturales, su espacio cognitivo, sin perder de vista los mecanismos de comprensión que maneja ante los estímulos que se le proporcionan, porque el código que interpretan no es sólo un código oral o escrito, sino toda la información que perciben sus sentidos en el contexto cultural en el que se desarrollan.

Otra manera de facilitar el aprendizaje de la lengua, es por medio de la competencia estratégica, la cual supone la capacidad de movilizar los recursos necesarios para un aprendizaje y una comunicación eficaces. En este sentido, el aprendiz pasa de adoptar un papel prácticamente pasivo a ser el responsable de su propio aprendizaje a través de la reflexión sobre el proceso que le puede llevar el dominio de la lengua. Independientemente de la denominación de las estrategias (cognitivas, metacognitivas, socioafectivas, etc.) pueden aplicarse al aula a través de la reflexión en actividades habituales.

Las actividades de la lengua responden a un proceso de codificación y descodificación, además de inferencia. Para que esto tenga éxito es necesario incluir la enseñanza / aprendizaje de las cuatro destrezas lingüísticas (comprensión lectora, comprensión auditiva, expresión escrita y expresión oral), donde alternan la expresión, la comprensión y la mediación. A partir del capítulo 13 hasta el 16, se estudian los procesos, habilidades y estrategias de cada una de las destrezas, así como la didáctica del proceso educativo.

En primer lugar, la comprensión lectora parte de una estrategia denominada bottom up, centrada en la descodificación, frente a otra que se centra más en la inferencia top down, hasta llegar a una posición interactiva que integra ambos modelos. Un buen punto de referencia para aplicar tipos de lectura con los estudiantes es la revisión de las escalas ilustrativas que expone el Marco Común Europeo de Referencia para las Lenguas (MCER: 2002) pues describe qué puede hacer un aprendiz de acuerdo con el nivel que puede alcanzar, según sea lectura extensiva o detallada. En segundo lugar, estos procesos de codificación y descodificación también se aplican en la destreza de la comprensión auditiva. A diferencia de la comprensión lectora, al escuchar, el estudiante no tiene control en absoluto sobre el texto oral.

Una vez que se conocen las dificultades del alumno es necesario tomar en cuenta la manera de procesar la información, las distintas formas de escuchar un texto, las técnicas que permitan una lectura extensiva o intensiva, los requisitos que deben tener los tipos de textos que contribuyen a la comprensión auditiva y en general, la secuencia didáctica para alcanzar el objetivo en el aula al desarrollar esta habilidad. Todo proceso de planificación que tome en cuenta la teoría de la comunicación en cuanto a la codificación y la elaboración del discurso, permitirá integrar simultáneamente las destrezas receptivas (comprensión auditiva y comprensión lectora) y las destrezas productivas (expresión escrita y oral) para convertirse en actividades de interacción o mediación.

El capítulo 17 se refiere a la importancia de integrar las destrezas en la comunicación del aula para que sea más sencillo poder usarlas en un contexto real. El MCER, el Plan Curricu- 
lar del Instituto Cervantes (PCIC) o los estándares de la American Council on the Teaching of Foreign Languages (ACTFL) son documentos que representan una buena muestra del esfuerzo por taxonomizar el proceso gradual de conocimiento de una lengua y que resultan imprescindibles para la programación de la enseñanza y la evaluación del aprendizaje.

Por otro lado, en el capítulo 18 se hace alusión a la historia de la metodología de la enseñanza de lenguas, la cual ha sido sistematizada y descrita de manera detallada en anteriores trabajos. Desde finales del siglo XIX hasta nuestros días han ido surgiendo una serie de métodos y enfoques, los cuales se mencionan en el capítulo 19: método gramática-traducción, método directo, enfoque oral, enfoque situacional, método audiolingual, nocional-funcional, enfoque por tareas, sugestopedia, aprendizaje comunitario, respuesta física total, aprendizaje integrado de contenidos y lenguas extranjeras (AICLE). Estos métodos y enfoques son uno de los factores prioritarios por considerar cuando se prepara una clase de español como segunda lengua. Otros son: el contexto en el que se desarrolla el aprendizaje, el centro en el que se imparte la docencia o los objetivos fundamentales que persiguen los aprendices en cuanto a contenidos o destrezas. En fin, la programación de un curso de español L2/LE debe seguir una serie de pautas con el fin de completar un diseño que contemple desde el propio planteamiento metodológico de la programación hasta la evaluación final. De hecho, en el capítulo 20 se ofrecen tablas con la programación de unidades didácticas que contemplan estos aspectos. El apartado expone ejemplos de cómo deben plantearse los objetivos, las actividades, contenidos por destrezas lingüísticas y ejercicios del enfoque por tareas.

Para lograr el alcance de los objetivos de la clase, el docente debe preocuparse por seleccionar los recursos didácticos idóneos, de ahí que el capítulo siguiente muestre los modelos y ejercicios que conducen a las actividades comunicativas. Un ejemplo que aparece es el de las páginas web, las cuales ofrecen no solo compendios de recursos bibliográficos para la clase de español como LE/LE, sino también infinidad de recursos didácticos elaborados, prácticas y actividades hechas que se pueden llevar al aula simplemente con su respectiva descarga.

No se podía dejar de lado la referencia a las plataformas como entornos virtuales de aprendizaje, a través de las cuales se crean cursos online que mantienen una comunicación sincrónica (chats, mensajes, videoconferencias) como asincrónica (correos, foros). Como se sabe, las posibilidades de uso de internet se amplían con las opciones de compartir la información que ofrecen los blogs y redes sociales: Twiter, Instragram, Whastapp, Facebook. O bien, a través de pizarras electrónicas se pueden preparar clases más visuales, con material interactivo.

Los autores dejan claro que a pesar de poder contar con recursos que provoquen la interacción en el aula, esto en realidad depende de un conjunto de factores: número de estudiantes, nivel de dominio, características, actitud y motivación del profesor y alumnos, la relación entre los educandos, el tiempo de duración de la clase, la disposición del mobiliario, la metodología usada, entre otros. Todos estos factores van a contribuir también al proceso evaluativo del curso y a los resultados que se obtengan. La evaluación se produce dentro de la clase, pero también fuera de ella, es decir, se determina el grado de conocimiento en relación con los contenidos y destrezas trabajados, y se pone en relación con el dominio "objetivo" de la lengua que tiene y que puede ser certificado a efectos académicos, profesionales, etc. 
Ya en el capítulo 24 se exponen los tipos de evaluación que existen: formativa, sumativa, del proceso, de resultados, cualitativa, cuantitativa y subjetivo/objetiva. Asimismo, los distintos procedimientos de evaluación en el aula: listas de control, hojas de seguimiento, hojas de observación, diario de clase, tutorías, cuestionarios, trabajo final, portafolio o dossier, entre otros. Al final de la lectura podemos inferir que al crear una herramienta útil de medición representa seguir una serie de pasos de forma rigurosa para obtener un resultado válido, fiable y viable. No es sencillo, por eso se ofrecen ejemplos de cómo realizar este tipo de instrumentos para cada una de las destrezas lingüísticas.

Finalmente, pero no menos importante, el capítulo número 25 titulado: "El español con fines especificos", nombre que surge por las exigencias de la demanda lingüística y para adaptarse a la realidad de la enseñanza del español como L2/LE; de esta manera, se reconoce la interconexión que hay en muchas ocasiones entre la lengua general y la lengua de especialidad. Se consideran lenguas de especificidad en el ámbito del español como L2/ LE las lenguas de las ciencias, la técnica y las profesiones. Cada una de las lenguas posee unos recursos específicos, fundamentalmente léxicos, aunque también sintácticos, estilísticos, pragmáticos y funcionales que se utilizan en condiciones comunicativas consideradas especializadas.

Este libro expone en su cierre las soluciones a todas las preguntas que se plantean en cada capítulo, lo cual permite al lector repasar los conceptos, actualizarse y refrescar cada uno de los temas que aquí se concitan. Con total certeza se puede afirmar que es una obra dedicada a la formación de profesores de español L2/LE, muy útil para estructurar la experiencia docente, además de muy clara, pues permite conocer los trabajos e investigaciones realizadas a través de la historia hasta la actualidad; de la teoría se pasa a ejemplos concretos y de ahí a cómo aplicarlos según el contexto. Cada persona que lea este manual se sentirá identificado de una u otra forma con la aplicación de un enfoque, la puesta en práctica de una teoría o método, con su vivencia en el aula, o bien, con la planificación de su clase. Un texto de esta naturaleza permite a los docentes concentrar los esfuerzos en el aula en aquellos aspectos que se consideren de especial relevancia en función de las necesidades del curso. 